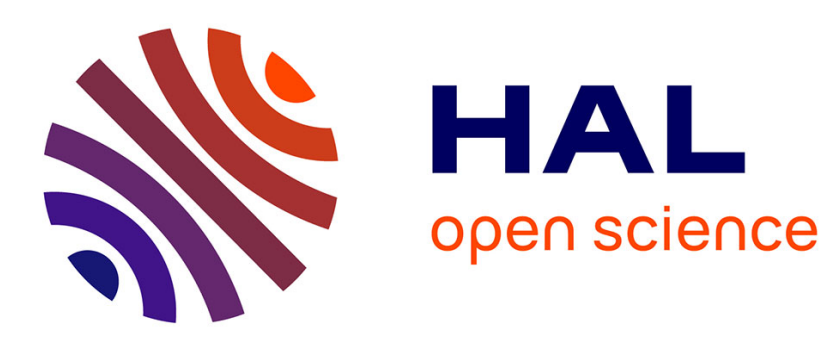

\title{
Reliability of Large-Eddy Simulations: Benchmarking and Uncertainty Quantification
}

\author{
Maria-Vittoria Salvetti, Marcello Meldi, Luca Bruno, Pierre Sagaut
}

\section{To cite this version:}

Maria-Vittoria Salvetti, Marcello Meldi, Luca Bruno, Pierre Sagaut. Reliability of Large-Eddy Simulations: Benchmarking and Uncertainty Quantification. Direct and Large-Eddy Simulation X, 24, pp.15-23, 2018. hal-02115804

\section{HAL Id: hal-02115804 https://hal-amu.archives-ouvertes.fr/hal-02115804}

Submitted on 30 Apr 2019

HAL is a multi-disciplinary open access archive for the deposit and dissemination of scientific research documents, whether they are published or not. The documents may come from teaching and research institutions in France or abroad, or from public or private research centers.
L'archive ouverte pluridisciplinaire HAL, est destinée au dépôt et à la diffusion de documents scientifiques de niveau recherche, publiés ou non, émanant des établissements d'enseignement et de recherche français ou étrangers, des laboratoires publics ou privés. 


\title{
Reliability of Large-Eddy Simulations: Benchmarking and Uncertainty Quantification
}

\author{
M.V. Salvetti, M. Meldi, L. Bruno and P. Sagaut
}

\section{Assessment of Quality and Reliability of Large-Eddy Simulations}

Nowadays, large-eddy simulation (LES) is increasingly applied to complex flow configurations of interest in technological or environmental applications. In this context, the assessment of the quality and reliability of LES results has become a topic of increasing interest. For LES this task is particularly difficult, because different sources of uncertainty may have comparable effects and may interact in a nonlinear way leading to counterintuitive results. A typical example is the interaction between discretization errors and subgrid scale (SGS) modeling. The related unexpected result behaviors observed in the literature are the following: (i) for given scheme and SGS model, accuracy deteriorating with grid refinement, (ii) for given grid and SGS modeling, lower-order schemes giving better results than higher-order ones or, (iii) for given grid and numerical scheme, no model simulations giving better results than LES with SGS modeling (see e.g. [1,2]). The strategies proposed in the literature to manage numerical errors are rather controversial. A first way of thinking is that numerical errors should be made negligible and all the burden should be on the SGS model. This can be achieved either by using high-order not-dissipative schemes, as recommended e.g. in [3, 4], or by applying explicit filtering of width significantly larger than the grid size $[5,6]$. However, due to the huge computational resources

\author{
M.V. Salvetti $(\varangle)$ \\ Dipartimento di Ingegneria Civile e Industriale, University of Pisa, Pisa, Italy \\ e-mail: mv.salvetti@ing.unipi.it \\ M. Meldi \\ PPRIME, CNRS-University of Poitiers-ENSMA, Poitiers, France \\ L. Bruno \\ Dipartimento di Architettura e Design, Politecnico di Torino, Turin, Italy \\ P. Sagaut \\ M2P2, Aix Marseille University, CNRS, Marseille, France
}


required, both these strategies are unpractical for complex flows of practical interest. A completely different approach is to get rid of physically based SGS models and to use the dissipation provided by numerical schemes both for numerical stabilization and SGS modeling (see, e.g., [7, 8]). Although this latter strategy may be attractive from a practical viewpoint, since it eliminates the need of implementing a SGS model, the quality of the results strongly depend on the characteristics of the used numerical methods, which should have again a high-order of accuracy and provide a dissipation concentrated on the smallest resolved scales. Numerical schemes usually available in commercial and open-source codes may conversely lead to inaccurate results due to a too strong numerical damping (see, e.g., [9]). As a consequence, the most widely adopted practice is to use an explicit SGS model and to accept a not-perfect numerics. In this context, the importance of different errors and their interactions should be assessed.

Benchmarks and comparisons with reference data, either from experiments or direct numerical simulations, have classically been used in the last decades for appraising the accuracy of LES results and the suitability of numerical methods or SGS models. However, uncertainties can also derive from a lack of knowledge of the considered problem set-up, as for instance some features of the incoming flow which are difficult to be perfectly controlled and/or characterized in experiments. Another critical issue is indeed the sensitivity of LES results to the different simulation parameters. Systematic sensitivity studies are difficult to be carried out because of the large cost of each single LES simulation.

Methodologies aimed at obtaining insights in the error behavior and interaction and in the sensitivity to the parameters of the LES results have been recently proposed. One is the error-landscape approach (see, e.g., [10]) in which a full response surface of the LES error behavior is built from a systematic variation of influencing parameters, as, e.g., model constants and grid resolution. This approach provides a framework to characterize the combined effects of modeling and discretization, but at the cost of a large number of simulations, which may become unaffordable for complex cases or when a large number of parameters is involved. Another approach, which is being increasingly used in recent years in computational fluid dynamics, is Uncertainty Quantification (UQ), in which the uncertain or unknown parameters are modeled as input random variables with a given probability distribution. These uncertainties can be propagated through the computational model to statistically quantify their effect on the results. Since this propagation process implies large computational costs, especially for LES, a computationally inexpensive surrogate model is usually adopted to build continuous response surfaces in the parameter space starting from a few deterministic simulations. Different techniques exist to build these surrogate models, such as, for instance, generalized Polynomial Chaos, Stochastic Collocation or Kriging. A few specific applications of stochastic UQ approaches to LES can be found in the recent literature [11-16].

The paper is further organized as follows. In Sect. 2 a few examples of benchmarks involving LES simulations are presented, to highlight the kind of useful indications but also the limits of this approach. In Sect. 3 an example of stochastic UQ for LES, based on generalized Polynomial chaos (gPC), is given. 


\section{Benchmarking: A Few Examples}

Benchmarks are commonly used to assess the accuracy and reliability of CFD results. Usually, there is a reference experiment or DNS and different numerical results are collected and compared against the reference data. Several benchmarks are documented in the literature also involving LES.

As an example, among the oldest ones is the benchmark on the flow around a square cylinder at $\mathrm{Re}=22,000$ (see the review in [17] and the ERCOFTAC Wiki database, http://uriah.dedi.melbourne.co.uk/). RANS and LES results were compared against reference experimental data in terms of aerodynamic loads and of mean and fluctuating velocity fields. It was concluded in [17] that all in all LES seems to give better predictions than RANS and it better captures the complex features of the flow. However, none of the LES results were found to be uniformly good and entirely satisfactory and there were large differences between the individual calculations which were not completely explained. Several possible reasons for the lack of agreement of LES results with the experiments were indicated, such as insufficient grid resolution near the side walls of the cylinder and/or in the spanwise direction, neglect of turbulence in the incoming stream, numerical diffusion and insufficient domain extent.

Benchmark results are even more difficult to be analyzed for benchmarks in which a-priori there are no reference experiments or simulations, as in the case of the BARC benchmark, dealing with the flow around a rectangular cylinder with chord-to-depth ratio equal to 5 and infinite spanwise length, recently reviewed in [18]. This configuration is of practical interest for civil and industrial structures; in spite of the simple geometry, the related flow dynamics and topology is complex, being turbulent with separation from the upstream corners and reattachment on the cylinder side and vortex shedding from the rear corners. Besides comparing experimental and numerical results, one of the goals of BARC is to assess the consistency of wind tunnel measurements carried out in different facilities and of computational results obtained through different approaches. About 20 wind tunnel tests and 50 numerical simulations were reviewed in [18]. LES simulations amount to more than $50 \%$ of the collected numerical contributions to BARC; therefore, this context gives also a good opportunity for the assessment and the validation of results obtained through LES codes. The LES contributions to BARC were specifically reviewed in [19]. For some quantities an overall very good agreement was found between the different numerical results and experimental data. An example is the mean drag coefficient, $\left\langle C_{D}\right\rangle$, i.e. the non-dimensionalized drag acting on the cylinder averaged in time and in the spanwise direction. Only one measurement of this quantity is available within BARC, giving $\left\langle C_{D}\right\rangle=1.03$; previous experiments also indicate that the mean drag coefficient is very close to one. The predictions of the numerical contributions to BARC are summarized in Table 1. It can be seen that the ensemble average of the values given by LES simulations and that of the predictions of URANS and hybrid approaches are practically the same and very close to the experimental value. However, rather unexpectedly, it appears that the dispersion of the LES predictions is 
Table 1 Mean drag coefficient: BARC numerical results

\begin{tabular}{l|l|l|l}
\hline & LES & Hybrid and URANS & Selected LES \\
\hline Ensemble average & 1.07 & 1.07 & 0.99 \\
\hline Range & $0.96-1.39$ & $0.96-1.29$ & $0.96-1.04$ \\
\hline
\end{tabular}

larger than that of URANS and hybrid URANS/LES simulations. Nonetheless, it turns out that the largest values of $\left\langle C_{D}\right\rangle$ were obtained in a 2D LES and in 3D LES having a too small computational domain; if these simulations are discarded, the dispersion range of LES predictions becomes smaller than that of URANS and hybrid simulations (see the last column of Table 1). Therefore, it appears that wrong choices of the computational domain dimensions in LES can lead to very bad predictions, worse than those of URANS, independently of the other numerical and modeling ingredients. This gives an example of best practice indications that can be obtained from benchmarks. On the other hand, for other quantities, such as the pressure distribution over the cylinder lateral surface or the amplitude of time oscillations of lift, the dispersion of both experimental and numerical results is so large that it would be unacceptable for engineering or design applications. As an example, Fig. 1 summarizes the ensemble statistics of standard deviation in time of the pressure coefficient on the lateral cylinder side; the range of values obtained in experiments and in LES and detached-eddy (DES) simulations is reported for different locations over the cylinder lateral side, together with the median, the 25-th and the 75-th percentile values computed among all the contributions. LES and DES give a distribution qualitatively similar to the experiments but their dispersion is larger than in experiments. Different possible reasons of the observed large dispersion were analyzed in [18], but not conclusive remarks could be drawn. It is certain that some flow features of the BARC are extremely sensitive to small uncertainties, which may be present both in wind-tunnel tests and in computations. Sources of uncertainties are different in experiments and in simulations, further complicating their comparison. Recent stochastic UQ and sensitivity studies for URANS and LES of the BARC flow can be found in $[20,21]$ (not shown here for the sake of brevity).

\section{Uncertainty Quantification Through Generalized Polynomial Chaos}

The polynomial chaos approach is a spectral decomposition of random processes in terms of an orthogonal basis. The uncertain parameters are assumed to be random variables, $\xi(\omega)$ and the output quantities, $E(\omega)$, also considered as random fields, are approximated through their Galerkin projection over a polynomial orthogonal basis: $E(\omega)=\sum_{k=0}^{\infty} a_{k} \xi(\omega)$. In gPC, the response surface of the output quantities in the parameter space is obtained by truncating the sum to a finite (small) number, $N$. The polynomial family is chosen by the user, on the basis of the (assumed) stochastic 

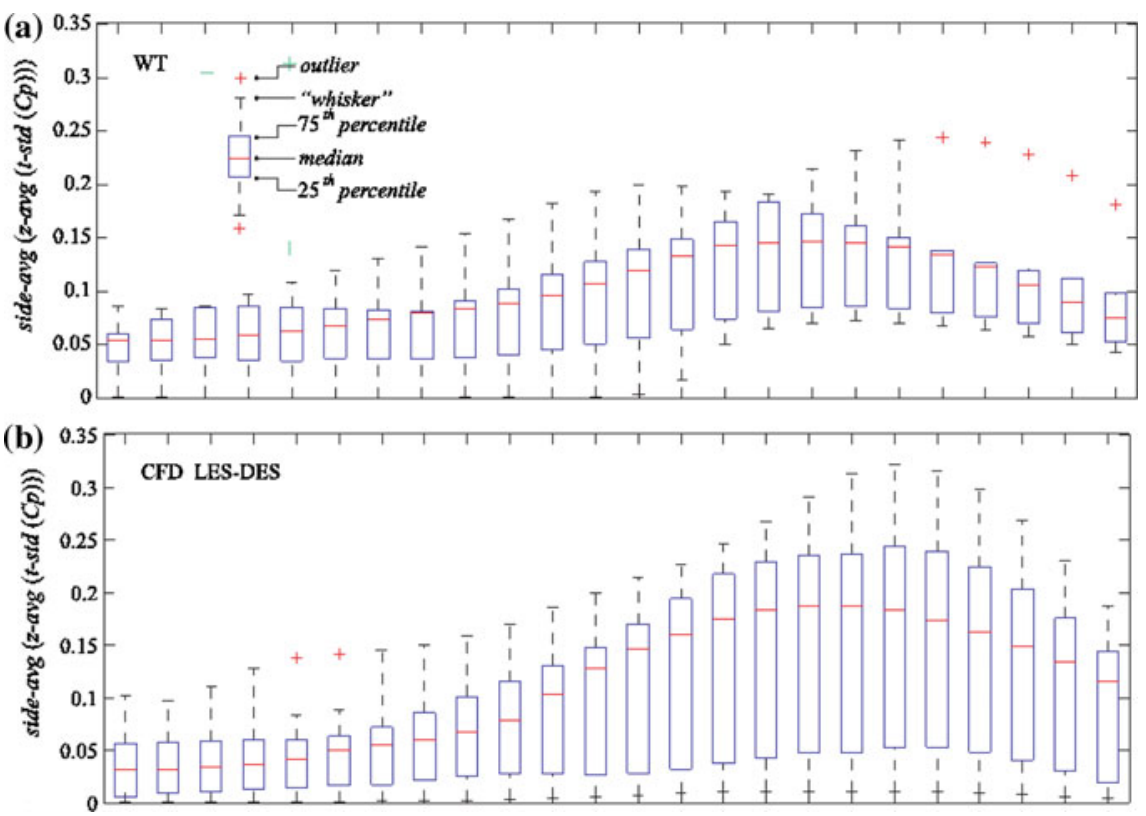

Fig. 1 Ensemble statistics of the time standard deviation of the pressure coefficient distribution over the cylinder side; a experiments, b LES and DES contributions. Taken from [18]

properties of the input parameters. This choice affects the speed of convergence of the truncated series. Each coefficient of the truncated series can be computed as follows: $a_{k}=\frac{\left\langle E, \phi_{k}\right\rangle}{\left\langle\phi_{k}, \phi_{k}\right\rangle}$, where $\langle\cdot, \cdot\rangle$ is a suitable scalar product. The number of points used to discretize the random variable space, which corresponds to the number of deterministic simulations to be carried out, is chosen in order to obtain converged integrals when computing the polynomial coefficients. Each deterministic simulation corresponds to a quadrature point in the parameter space.

As an example of error quantification in LES through $\mathrm{gPC}$, we consider a spatially evolving mixing layer at a Reynolds number, based on the difference between the two stream velocities and on the vorticity thickness at the inlet, equal to 700. A highly-resolved LES (HRLES) was carried out by using the dynamic Smagorinsky SGS model; the results were validated against DNS and LES data in the literature and are used as a reference for the UQ of errors in LES carried out on grids having a resolution four time coarser than the HRLES one in the streamwise direction and twice coarser in the other directions and using the standard Smagorinsky SGS model. Further details on the test case and on the simulation set up can be found in [14]. The sensitivity of coarse LES results to the following parameters is investigated: the stretching ratio of the grid cells in the streamwise and lateral directions and the Smagorinsky model coefficient. Although these parameters can obviously be fixed to precise deterministic values, the lack of knowledge of their appropriate values 
can be considered as an epistemic uncertainty. They are then considered as uncertain parameters and the previously described gPC approach is used for UQ. Their probability density function (PDF) is assumed here to be uniform over the considered variation range (see [14]) and, hence, Legendre polynomials are considered in the gPC expansion, which is truncated to $N=3 ; 64$ coarse LES were then carried out to build the response surface in the parameter space. Uncertainty propagation on the errors for three quantities of practical interest, viz. the mean streamwise velocity, the momentum thickness and the shear stress, is investigated. The error was computed as the $L_{2}$ norm of the difference between the values of the above quantities in HRLES and those in coarse LES, for different streamwise sections, averaging over the $y$ and $z$ directions. A typical feature of the considered spatially evolving flow is the progressive transition from a laminar regime, highly dependent on the inlet conditions, to a fully-turbulent one. The computational domain can therefore be divided in two zones, by assuming that the fully-turbulent region starts at the location where the signature of the inlet perturbations on the flow is no more apparent, roughly at $x=12 \Lambda, \Lambda$ being the adopted reference length, multiple of the inlet vorticity thickness. As an example of the kind of information which can be obtained from such an analysis, Fig. 2a shows the behavior of the mean stochastic error for the considered quantities of interest, at different distances from the domain inlet. It is clear that error on the mean streamwise velocity (circles) is much lower than that on the other two variables, never exceeding $1.5 \%$. The mean errors on the momentum thickness and on the shear stress significantly vary with the distance from the domain inlet. In the inlet-dependent zone, the stochastic error decreases moving downstream. This indicates that the most critical part of this region is connected with the initial stages of transition. The opposite behavior of the error is observed in the turbulent region; this is probably due to a progressively coarsening of the streamwise resolution when moving downstream (the grid nodes are clustered near the inlet) and to the pres-

(a)

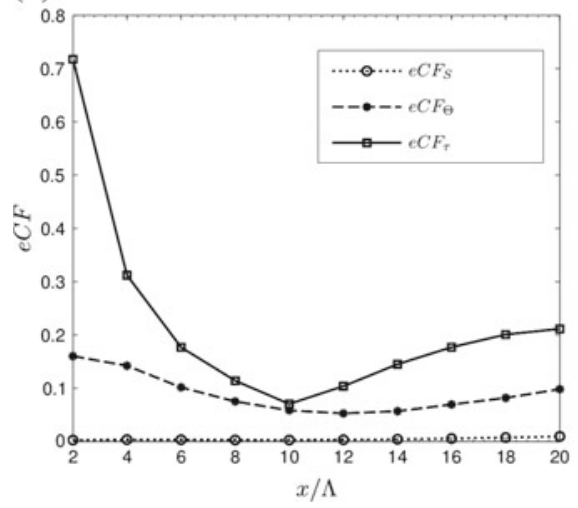

(b)

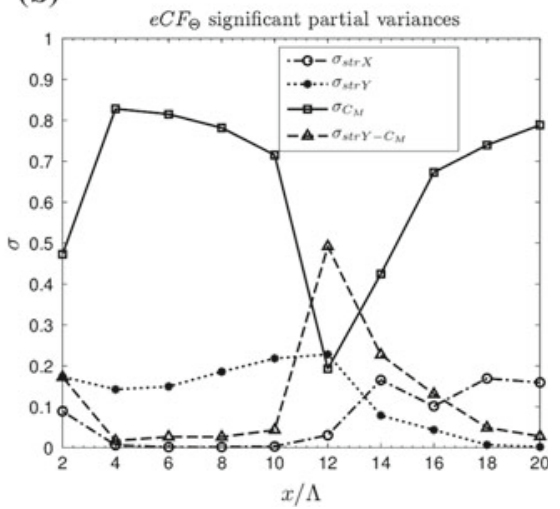

Fig. 2 a Mean stochastic error for different output quantities. b Partial variances for the error on momentum thickness. Taken from [14] 

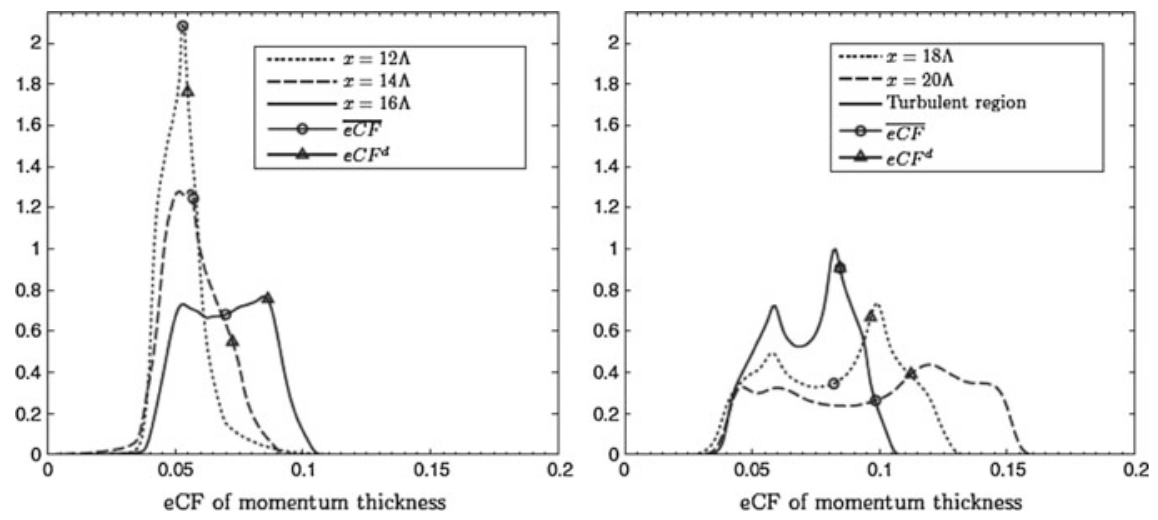

Fig. 3 PDF of the error on the momentum thickness in the inlet-dependent zone (left panel) and in the fully-turbulent one (right panel). Open circles mean stochastic value of the error; triangles error obtained in deterministic simulations carried out with average values of the input parameters. Taken from [14]

ence of progressively smaller turbulent flow scales. Partial variances, which quantify the sensitivity of the errors to the single parameters and to their interactions, can also be computed and analyzed. An example is given in Fig. $2 \mathrm{~b}$ showing the partial variances for the error on the momentum thickness. It can be seen that the value of the Smagorinsky constant has the largest impact on the error; however, a peculiar behavior is observed near the border between the inlet-dependent and fully-turbulent zones with strong coupling between the effects of the Smagorinsky constant and of the grid stretching in the lateral direction. Further information on the error behavior can be obtained from the error PDFs, as shown for instance in Fig. 3 again for the momentum thickness. In the inlet-dependent region, a huge number of parameter combinations give error values close to the most probable one (the peak in the PDF), but the error may be significantly larger for some combinations of the parameters. Conversely, in the fully-turbulent region, the error distribution is less clustered than in the inlet-dependent zone, and, hence, the stochastic mean error value (open circles) can be significantly different from the most probable one. In both zones, both the stochastic mean and most probable error values are in turn different from the errors obtained in deterministic LES carried out with a set of average parameter values in the considered range. For a more detailed analysis we refer to [14], where an optimization of the parameters based on the built error response surface can also be found.

Summarizing, assessment of the accuracy and reliability of LES results, especially for complex applications, is still today a challenge. Benchmarks can highlight clear trends, but the reasons of observed dispersion can not always be explained, due to different uncertainty sources present both in experiments and simulations. Uncertainty quantification techniques can be useful to give hints on the error dynamics and on the reliability of LES results. However, also UQ techniques become not viable 
for large numbers of uncertain parameters (the so-called 'curse of dimensionality'). A huge research effort is indeed currently made by the UQ scientific community to develop more efficient techniques to overcome this problem.

\section{References}

1. Vreman, B., Geurts, B.J., Kuerten, H.: Comparison of numerical schemes in large-eddy simulations of the temporal mixing layer. Int. J. Numer. Methods Fluids 22, 297-311 (1996)

2. Ouvrard, H., Koobus, B., Dervieux, A., Salvetti, M.V.: Classical and variational multiscale LES of the flow around a circular cylinder on unstructured grids. Comput. Fluids 39, 1083-1094 (2010)

3. Ghosal, S.: An analysis of numerical errors in large-eddy simulations of turbulence. J. Comput. Phys. 125, 187-206 (1996)

4. Kravchenko, A.G., Moin, P.: On the effect of numerical errors in large eddy simulations of turbulent flows. J. Comput. Phys. 131, 310-322 (1997)

5. Geurts, B.J., van der Bos, F.: Numerically induced high-pass dynamics in large-eddy simulation. Phys. Fluids 17, 125103 (2005)

6. Bose, S.T., Moin, P., You, D.: Grid-independent large-eddy simulation using explicit filtering. Phys. Fluids 22, 105103 (2010)

7. Boris, J.P., Grinstein, F.F., Oran, E.S., Kolbe, R.L.: New insights into large eddy simulation. Fluid Dyn. Res. 10, 199-228 (1992)

8. Karamanos, G.S., Karniadakis, G.E.: A spectral vanishing viscosity method for large-eddy simulations. J. Comput. Phys. 163, 22-50 (2000)

9. Garnier, E., Mossi, M., Sagaut, P., Comte, P., Deville, M.: A spectral vanishing viscosity method for large-eddy simulations. J. Comput. Phys. 163, 22-50 (2000)

10. Meyers, J., Geurts, B.J., Baelmans, M.: Database analysis of errors in large-eddy simulations. Phys. Fluids 15, 2740-2755 (2003)

11. Christophe, J., Moreau, S., Hamman, C.W., Witteveen, J.A.S., Iaccarino, G.: Uncertainty quantification for the trailing-edge noise of a controlled-diffusion airfoil. AIAA J. 53, 42-54 (2015)

12. Carnevale, M., Montomoli, F., D’Ammaro, A., Salvadori, S., Martelli, F.: Uncertainty quantification: a stochastic method for heat transfer prediction using LES. J. Turbomach. 135, 051021 (2013)

13. Congedo, P.M., Duprat, C., Balarac, G., Corre, C.: Numerical prediction of turbulent flows using Reynolds-averaged Navier-Stokes and large-eddy simulation with uncertain inflow conditions. Int. J. Numer. Methods Fluids 72, 341358 (2013)

14. Meldi, M., Salvetti, M.V., Sagaut, P.: Quantification of errors in large-eddy simulations of a spatially evolving mixing layer using polynomial chaos. Phys. Fluids 24, 035101 (2012)

15. Meldi, M., Lucor, D., Sagaut, P.: Is the Smagorinsky coefficient sensitive to uncertainty in the form of the energy spectrum? Phys. Fluids 23, 125109 (2011)

16. Lucor, D., Meyers, J., Sagaut, P.: Sensitivity analysis of large-eddy simulations to subgridscale-model parametric uncertainty using polynomial chaos. J. Fluid Mech. 585, 255-279 (2007)

17. Rodi, W.: Comparison of LES and RANS calculations of the flow around bluff bodies. J. Wind Eng. Ind. Aerodyn. 69-71, 55-75 (1997)

18. Bruno, L., Salvetti, M.V., Ricciardelli, F.: Benchmark on the aerodynamics of a rectangular 5:1 cylinder: an overview after the first four years of activity. J. Wind Eng. Ind. Aerodyn. 126, 87-106 (2014)

19. Salvetti, M.V., Bruno, L.: Reliability of LES simulations in the context of a benchmark on the aerodynamics of a rectangular 5:1 cylinder. In: Fröhlich, J., Kuerten, H., Geurts, B.J., Armenio, V. (eds.) Direct and Large-Eddy Simulation IX. ERCOFTAC Series, pp. 161-167. Springer, Berlin (2015) 
20. Mariotti, A., Salvetti, M.V., Shoebi-Omrani, P., Witteveen, J.A.S.: Stochastic analysis of the impact of freestream conditions on the aerodynamics of a rectangular 5:1 cylinder. Comput. Fluids 136, 170-192 (2016)

21. Mariotti, A., Siconolfi, L., Salvetti, M.V.: Stochastic sensitivity analysis of large-eddy simulation predictions of the flow around a 5:1 rectangular cylinder. Eur. J. Mech. B. Fluids 62, 149-165 (2017) 